\title{
Avaliação da qualidade de vida de pessoas vivendo com HIV/AIDS: revisão integrativa
}

\author{
Evaluating the quality of life of people living with HIV/AIDS: integrative review \\ Evaluación de la calidad de vida de personas que viven con VIH/SIDA: revisión integradora
}

\author{
Sérgio Corrêa Marques', Denize Cristina de Oliveira", Hellen Pollyanna Mantelo Cecilio"', \\ Cinthia Pereira Silvalv, Leonardo Alves Sampaiov ${ }^{v}$ Virgínia Xavier Pereira da Silva ${ }^{v}$
}

\begin{abstract}
RESUMO
Objetivo: analisar estudos referentes à avaliação da qualidade de vida de pessoas vivendo com HIV no Brasil, por meio de uma revisão integrativa da literatura. Método: trata-se de revisão integrativa que incluiu 22 artigos científicos publicados no período de 2011 a 2019. Resultados: os principais instrumentos utilizados na avaliação da qualidade de vida foram o WHOQOL-HIV bref e o HAT-QOL. Os domínios com maiores escores na avaliação foram confiança no médico, psicológico e espiritualidade; as piores avaliações relacionavam-se com os domínios meio ambiente e preocupação com o sigilo. A exposição do diagnóstico impacta negativamente para a qualidade de vida bem como as condições de vida e saúde. Considerações finais: a qualidade de vida é um construto multidimensional e subjetivo com implicação direta na saúde e tratamento das pessoas vivendo com HIV, portanto, compreender os fatores que impactam sua avaliação é primordial na elaboração de políticas públicas e melhores condições de vida. Descritores: Qualidade de Vida; Avaliação; Síndrome de Imunodeficiência Adquirida; HIV.
\end{abstract}

\section{ABSTRACT}

Objective: to analyze studies on evaluating the quality of life of people living with HIV in Brazil, by integrative literature review. Method: this integrative review included 22 scientific articles published from 2011 to 2019. Results: the instruments most used to assess quality of life were the WHOQOL-HIV bref and the HAT-QOL. The highest-scoring domains were trust in the doctor psychology and spirituality, while the lowest scores related to the environment and confidentiality concerns. Exposing the diagnosis has adverse impacts on both quality of life and conditions of life and health. Final considerations: quality of life is a multidimensional, subjective construct with direct implications for the health and treatment of people living with HIV. Accordingly, in order to develop public policies and improve conditions of life, it is essential to understand the factors that impact on its assessment.

Descriptors: Quality of Life; Evaluation Acquired Immunodeficiency Syndrome; HIV.

\section{RESUMEN}

Objetivo: analizar estudios sobre la evaluación de la calidad de vida de las personas que viven con el VIH en Brasil, mediante revisión integradora de la literatura. Método: esta revisión integradora incluyó 22 artículos científicos publicados entre 2011 y 2019. Resultados: los instrumentos más utilizados para evaluar la calidad de vida fueron el WHOQOL-HIV bref y el HAT-QOL. Los dominios de mayor puntuación fueron la confianza en el médico, la psicología y la espiritualidad, mientras que las puntuaciones más bajas se relacionaron con el medio ambiente y las preocupaciones de confidencialidad. Exponer el diagnóstico tiene impactos adversos tanto en la calidad de vida como en las condiciones de vida y salud. Consideraciones finales: la calidad de vida es un constructo subjetivo multidimensional con implicaciones directas para la salud y el tratamiento de las personas que viven con el VIH. En consecuencia, para desarrollar políticas públicas y mejorar las condiciones de vida, es fundamental conocer los factores que inciden en su evaluación.

Descriptores: Calidad de Vida; Evaluación; Síndrome de Inmunodeficiencia Adquirida; VIH.

\section{INTRODUÇÃO}

Estudos sobre HIV/AIDS revestem-se de significados pelas consequências e impactos mundiais da infecção, nos níveis político, econômico e social. Desde o início da epidemia até o fim de 2018,74,9 milhões de pessoas foram infectadas pelo HIV e estima-se que 37,9 milhões de pessoas vivem com HIV no mundo ${ }^{1}$. No Brasil, desde os primeiros casos até junho de 2019 foram registrados 966.058 casos, sendo 43.941 novos casos de infecção pelo HIV apenas em 2018, mantendo a média de 39.000 novos casos nos últimos cinco anos².

'Enfermeiro. Doutor. Professor Adjunto. Universidade do Estado do Rio de Janeiro. Rio de Janeiro, Brasil. E-mail: sergiocmarques@uol.com.br. ORCID: https://orcid.org/0000-0003-2597-4875

"Enfermeira. Doutora. Professora Titular. Universidade do Estado do Rio de Janeiro. Rio de Janeiro, Brasil. E-mail: dcuerj@gmail.com. ORCID: https://orcid.org/0000-0002-0830-0935 I'Enfermeira. Doutora. Professora Adjunta. Universidade Estadual de Maringá, Maringá, Brasil. E-mail: pollymantelo@gmail.com. ORCID: https://orcid.org/0000-0002-6597-432X IVEnfermeira. Mestre. Universidade do Estado do Rio de Janeiro. Rio de Janeiro, Brasil. E-mail: cinthiapereira22@hotmail.com. ORCID: https://orcid.org/0000-0002-1736-9512 VEnfermeiro. Mestre. Universidade do Estado do Rio de Janeiro. Rio de Janeiro, Brasil. E-mail: enfermeirolas@gmail.com. ORCID: https://orcid.org/0000-0001-8177-6385 VIEnfermeira. Mestre. Professora auxiliar. Centro Universitário Gama e Souza. Rio de Janeiro, Brasil. E-mail: vi-xavier@hotmail.com. ORCID: https://orcid.org/0000-0002-8267-9189 
A configuração atual da infecção pelo HIV demonstra um perfil de heterossexualização, feminilização, pauperização e interiorização da epidemia, associados intimamente com a profunda desigualdade social brasileira3. Apesar da magnitude da infecção no cenário nacional, observa-se a diminuição na taxa de detecção de AIDS - de 21,7/100.000 em 2012 para 17,8/100.000 em 2018 - a partir da orientação de tratamento precoce para todos infectados, independente da carga viral ${ }^{2}$.

Decorridas mais de três décadas de sua descoberta, o HIV/AIDS apresenta uma nova configuração clínica e epidemiológica, deixando no passado a associação com a presença da morte iminente e passando a figurar como uma doença crônica. É importante destacar o papel da terapia antirretroviral (TARV) para o aumento da expectativa de vida das pessoas vivendo com HIV e, consequentemente, para a mudança no olhar para essa condição de saúde ${ }^{4}$.

Apesar do reconhecimento de que o aumento do tempo de sobrevida influencia de forma positiva a vida das pessoas, entende-se que esse fator isoladamente não está relacionado diretamente com a melhoria na qualidade de vida (QV) das pessoas vivendo com HIV, uma vez que o diagnóstico implica em mudanças nos variados aspectos da vida, tais como sexuais, emocionais e sociais ${ }^{5,6}$

De acordo com a Organização Mundial de Saúde (OMS) a QV pode ser definida como uma "percepção do indivíduo sobre a sua posição na vida, dentro do contexto da cultura e no sistema de valores no qual ele vive e em relação a suas expectativas, seus padrões e suas preocupações" 7:28. Desta forma, identifica-se a QV das pessoas vivendo com HIV como um elemento multifatorial, associado aos aspectos funcionais, físicos, econômicos, emocionais, sociais, de espiritualidade, crenças pessoais e do meio ambiente, envolvendo a vivência subjetiva do HIV, assim como a atenção integral à saúde. Estudos desenvolvidos com a temática demonstram que os indivíduos encontram diferentes maneiras de adaptar e conviver com as mudanças ocorridas em sua vida, o que também influi na forma de avaliar a $Q V^{4,8,9}$.

É importante ressaltar que, na tentativa de compreensão da QV, deve-se tomar cuidado para não reduzir o conceito apenas aos fatores objetivos e subjetivos, já que este construto envolve as constantes inter-relações entre todos os elementos que compõem esse universo ${ }^{10}$.

Nesse contexto, considera-se relevante investigar o que as produções científicas apontam em relação à avaliação da QV das pessoas vivendo com HIV. Portanto, traçou-se como objetivo analisar estudos referentes à avaliação da qualidade de vida de pessoas vivendo com HIV no Brasil, por meio de uma revisão integrativa da literatura.

\section{MÉTODO}

Este estudo consiste em uma revisão integrativa, método que sintetiza informações disponíveis na literatura, de maneira sistemática e ordenada, visando construir um conjunto consistente de significados, capaz de relacionar achados empíricos e teóricos ${ }^{11}$. As etapas deste estudo foram desenvolvidas a partir das fases: identificação do tema; formulação da questão; levantamento dos estudos; seleção dos estudos; coleta de dados; avaliação dos estudos; análise dos dados; interpretação dos achados; discussão dos resultados; e, por fim, a apresentação da revisão integrativa ${ }^{11}$.

De forma a cumprir rigorosamente as etapas citadas, delimitou-se o tema central deste estudo como "avaliação da QV de pessoas vivendo com HIV" e a seguinte questão norteadora: qual a avaliação da QV das pessoas vivendo com HIV/AIDS no Brasil atualmente?

Para responder a questão, utilizou-se a Biblioteca Virtual em Saúde (BVS), selecionando como bases de dados o Banco de Dados em Enfermagem (BDENF), a Literatura Latino-Americana e do Caribe em Ciências da Saúde (LILACS), a Scientific Electronic Library Online (SCIELO) e a National Library of Medicine (PUBMED). As bases foram selecionadas na tentativa de captar todos os estudos publicados por pesquisadores brasileiros. Utilizou-se a combinação de descritores e operadores boleanos: "AIDS" OR "HIV" OR "síndrome da imunodeficiência adquirida" AND "qualidade de vida". Os critérios de inclusão foram artigos na íntegra, em português, disponíveis gratuitamente e indexados nas bases supracitadas. Como critério de exclusão, rejeitaram-se as dissertações e teses. O período de publicação elegido foi de 2011 a 2019, a fim de atualizar as informações sobre a temática. Foram analisados todos os artigos que atenderam os critérios de inclusão.

Para a organização das informações, foi elaborada uma planilha eletrônica no Microsoft Office Excel ${ }^{\circledR}$, contendo $^{\circ}$ as seguintes informações: título, autores, ano de publicação, periódico, objetivo, tipo de estudo, amostra, instrumento de avaliação da QV e resultados.

Após a avaliação dos estudos, por meio de um instrumento adaptado ${ }^{11}$, foram excluídos aqueles cujo delineamento da pesquisa não se adequava ao objetivo desta revisão bem como aqueles que apresentavam baixa qualidade metodológica. A análise dos estudos selecionados e a síntese dos dados extraídos dos artigos foram realizadas de forma descritiva, possibilitando observar, analisar, descrever e classificar os dados. 


\section{RESULTADOS}

A amostra inicial foi composta por 10.248 artigos encontrados nas bases de dados selecionadas. Ao inserir os critérios de inclusão previamente estabelecidos, o número de artigos foi reduzido para 166. Foram excluídas 69 publicações duplicadas entre as bases de dados e 37 por não possuírem relação com o objeto de estudo. Assim, 60 artigos tiveram seus resumos lidos e, após, 35 foram lidos na íntegra e 22 foram selecionados para análise e discussão. Os detalhes do processo de seleção dos estudos, conforme a recomendação PRISMA ${ }^{12}$, estão apresentados na Figura 1.

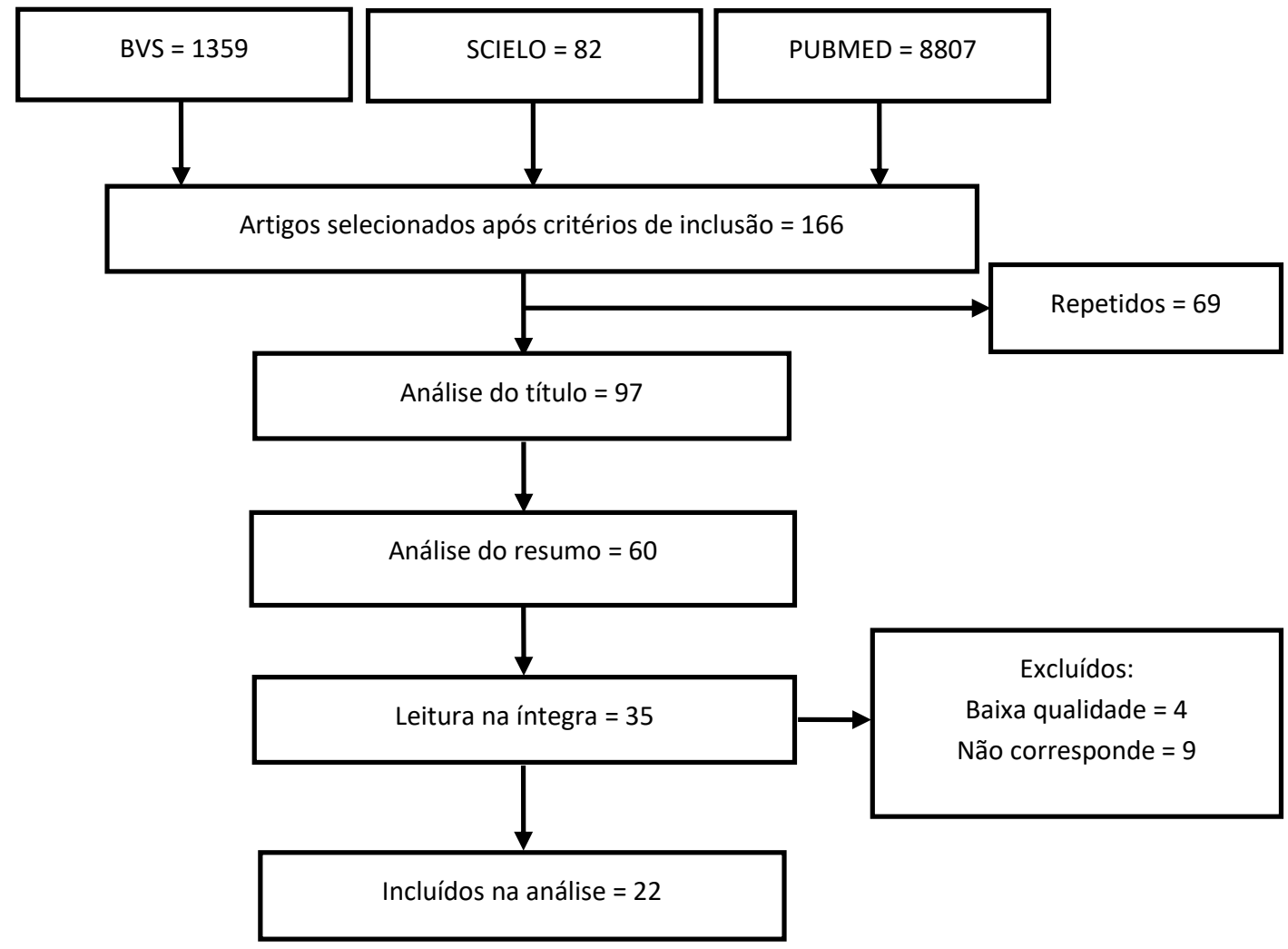

Figura 1: Seleção dos artigos nas bases de dados, 2011 a 2019. Rio de Janeiro, Brasil, 2020.

Entre os 22 artigos selecionados, 13 (59\%) foram publicados em revistas específicas da enfermagem, três (14\%) em revistas da psicologia e os demais, seis (27\%), em revistas médicas ou de saúde coletiva. Os artigos selecionados estão apresentados na Figura 2.

Sobre os instrumentos para avaliação da QV, 14 (64\%) artigos utilizaram o WHOQOL, sete (32\%) o HAT-QOL e um (4\%) artigo fez uma comparação entre o WHOQOL e o HAT-QOL, utilizando os dois instrumentos.

O HAT-QOL é composto por nove dimensões, sendo: confiança no médico; questões relativas à medicação; atividade geral; satisfação com a vida; conscientização HIV; atividade sexual; preocupação com a saúde; preocupação financeira; e, preocupação com sigilo ${ }^{5}$. O WHOQOL, por sua vez, é composto por seis domínios, sendo: físico; psicológico; nível de independência; relações sociais; meio ambiente; e, espiritualidade, religião e crenças pessoais $(E R C P)^{13}$. Ao analisar os dados socioeconômicos dos estudos selecionados, ressalta-se nas amostras, a predominância da baixa renda e menor escolaridade entre os participantes relacionando-se aos menores escores de $\mathrm{QV}^{5,13-16,20}$. Observa-se estreita associação entre tais variáveis e os domínios da QV meio ambiente, psicológico, preocupação com a saúde e tratamento médico.

Outra variável importante para análise da QV foi o sexo. Alguns estudos tiveram na composição da amostra apenas mulheres $^{13,21,26}$ ou apenas homens ${ }^{25}$; outros, pesquisaram mulheres e homens de forma aleatória ${ }^{5,14-20,22-24,26-32}$. Os estudos referem os menores escores de QV para mulheres e, a menor escolaridade, a menor renda, a dependência econômica e emocional do parceiro, a dificuldade na negociação do uso do preservativo, o medo da rejeição, a violência sexual e a ausência de autonomia sobre o próprio corpo configuram-se como os principais preditores para esse grupo $^{13,15,30}$ 


\begin{tabular}{|c|c|c|c|}
\hline Autor, ano & Título & $\begin{array}{l}\text { Instrumento/ } \\
\text { Amostra }\end{array}$ & $\begin{array}{l}\text { Estado da coleta } \\
\text { de dados }\end{array}$ \\
\hline REIS et al, $2011^{5}$ & $\begin{array}{l}\text { Qualidade de vida, aspectos sociodemográficos e de sexualidade de } \\
\text { pessoas vivendo com HIV/aids }\end{array}$ & $\begin{array}{l}\text { HAT-QOL } \\
229\end{array}$ & São Paulo \\
\hline $\begin{array}{l}\text { GASPAR et al, } \\
20111^{3}\end{array}$ & $\begin{array}{l}\text { Qualidade de vida de mulheres vivendo com o HIV/aids de um município } \\
\text { do interior paulista }\end{array}$ & $\begin{array}{l}\text { WHOQOL } \\
106\end{array}$ & São Paulo \\
\hline $\begin{array}{l}\text { LOPES et al, } \\
20111^{4}\end{array}$ & Qualidade de vida dos pacientes HIV positivo com mais de 50 anos & $\begin{array}{l}\text { HAT-QOL } \\
86\end{array}$ & Rio Grande do Sul \\
\hline $\begin{array}{l}\text { FERREIRA et al, } \\
20121^{5}\end{array}$ & $\begin{array}{l}\text { Qualidade de vida de portadores de HIV/AIDS e sua relação com linfócitos } \\
\text { CD4+, carga viral e tempo de diagnóstico }\end{array}$ & $\begin{array}{l}\text { WHOQOL } \\
205\end{array}$ & Minas Gerais \\
\hline $\begin{array}{l}\text { MEDEIROS et al, } \\
201216\end{array}$ & Religiosidade e qualidade de vida em pessoas com HIV & $\begin{array}{l}\text { WHOQOL } \\
90\end{array}$ & Paraíba \\
\hline $\begin{array}{l}\text { SILVA et al, } \\
2013^{3}\end{array}$ & $\begin{array}{l}\text { Qualidade de vida no contexto do HIV/aids: um estudo comparativo com a } \\
\text { população em geral }\end{array}$ & $\begin{array}{l}\text { WHOQOL } \\
561 \text { sem HIV } \\
286 \text { com HIV }\end{array}$ & Paraíba \\
\hline $\begin{array}{l}\text { MEDEIROS et al, } \\
20131^{7}\end{array}$ & $\begin{array}{l}\text { Determinantes biopsicossociais que predizem qualidade de vida em } \\
\text { pessoas que vivem com HIV/AIDS }\end{array}$ & $\begin{array}{l}\text { WHOQOL } \\
90\end{array}$ & Paraíba \\
\hline $\begin{array}{l}\text { BORBA et al, } \\
20141^{8}\end{array}$ & $\begin{array}{l}\text { Estudo comparativo do desempenho do WHOQOL-HIV bref e do HIV/aids } \\
\text { - target quality of life na avaliação de qualidade de vida de indivíduos que } \\
\text { vivem com HIV/aids }\end{array}$ & $\begin{array}{l}\text { HAT-QOL e } \\
\text { WHOQOL } \\
60\end{array}$ & Santa Catarina \\
\hline $\begin{array}{l}\text { OLIVEIRA FILHO } \\
\text { et al, } 20141^{9}\end{array}$ & Qualidade de vida de pessoas vivendo com HIV/aids & $\begin{array}{l}\text { WHOQOL } \\
30\end{array}$ & Paraíba \\
\hline $\begin{array}{l}\text { OKUNO et al, } \\
20142^{\circ}\end{array}$ & Qualidade de vida de pacientes idosos vivendo com HIV/aids & $\begin{array}{l}\text { HAT-QOL } \\
201\end{array}$ & São Paulo \\
\hline $\begin{array}{l}\text { BELLINI et al, } \\
20152^{1}\end{array}$ & Qualidade de vida de mulheres portadoras do HIV & $\begin{array}{l}\text { WHOQOL } \\
40\end{array}$ & São Paulo \\
\hline $\begin{array}{l}\text { GALVÃO et al, } \\
20152^{2}\end{array}$ & $\begin{array}{l}\text { Qualidade de vida e adesão à medicação antirretroviral em pessoas com } \\
\text { HIV }\end{array}$ & $\begin{array}{l}\text { HAT-QOL } \\
45\end{array}$ & Ceará \\
\hline $\begin{array}{l}\text { OLIVEIRA et al, } \\
20152^{3}\end{array}$ & Qualidade de vida e fatores associados em pessoas vivendo com HIV/aids & $\begin{array}{l}\text { WHOQOL } \\
146\end{array}$ & Piauí \\
\hline $\begin{array}{l}\text { OKUNO et al, } \\
20152^{4}\end{array}$ & $\begin{array}{l}\text { Qualidade de vida, perfil socioeconômico, conhecimento e atitude sobre } \\
\text { sexualidade de "pessoas que vivem" com o Vírus da Imunodeficiência } \\
\text { Humana }\end{array}$ & $\begin{array}{l}\text { HAT-QOL } \\
201\end{array}$ & São Paulo \\
\hline $\begin{array}{l}\text { CUNHA et al, } \\
20152^{5}\end{array}$ & $\begin{array}{l}\text { Qualidade de vida de homens com aids e o modelo da determinação social } \\
\text { da saúde }\end{array}$ & $\begin{array}{l}\text { WHOQOL } \\
138\end{array}$ & Ceará \\
\hline $\begin{array}{l}\text { TEIXEIRA et al, } \\
20152^{6}\end{array}$ & Avaliação da qualidade de vida de mulheres vivendo com HIV & $\begin{array}{l}\text { WHOQOL } \\
41\end{array}$ & Paraná \\
\hline $\begin{array}{l}\text { SOARES et al, } \\
20152^{7}\end{array}$ & $\begin{array}{l}\text { Qualidade de vida de pessoas que vivem com HIV/aids assistidas no } \\
\text { serviço especializado em Vitória (ES), Brasil }\end{array}$ & $\begin{array}{l}\text { HAT-QOL } \\
177\end{array}$ & Espírito Santo \\
\hline $\begin{array}{l}\text { HIPOLITO et al, } \\
20172^{8}\end{array}$ & $\begin{array}{l}\text { Qualidade de vida de pessoas convivendo com HIV/aids: relação temporal, } \\
\text { sociodemográfica e perceptiva da saúde }\end{array}$ & $\begin{array}{l}\text { WHOQOL } \\
100\end{array}$ & Rio de Janeiro \\
\hline $\begin{array}{l}\text { CALIARI et al, } \\
20182^{9}\end{array}$ & $\begin{array}{l}\text { Qualidade de vida de idosos vivendo com HIV/aids em acompanhamento } \\
\text { ambulatorial }\end{array}$ & $\begin{array}{l}\text { HAT-QOL } \\
81\end{array}$ & Minas Gerais \\
\hline $\begin{array}{l}\text { CECILIO et al, } \\
20183^{\circ}\end{array}$ & Qualidade de vida de pessoas vivendo com HIV & $\begin{array}{l}\text { WHOQOL } \\
281\end{array}$ & Rio de Janeiro \\
\hline $\begin{array}{l}\text { SILVEIRA et al, } \\
20193^{1}\end{array}$ & $\begin{array}{l}\text { Propriedades psicométricas do WHOQOL-HIV bref para avaliação da } \\
\text { qualidade de vida }\end{array}$ & $\begin{array}{l}\text { WHOQOL } \\
226\end{array}$ & Minas Gerais \\
\hline $\begin{array}{l}\text { CECILIO et al, } \\
20193^{2}\end{array}$ & $\begin{array}{l}\text { Qualidade de vida de pessoas vivendo com HIV atendidas em serviços } \\
\text { públicos de saúde }\end{array}$ & $\begin{array}{l}\text { WHOQOL } \\
281\end{array}$ & Rio de Janeiro \\
\hline
\end{tabular}

FIGURA 2: Artigos selecionados para análise do estudo. Rio de Janeiro, Brasil, 2020.

Entre os sete artigos que utilizaram o HAT-QOL, os maiores escores apresentados foram nos domínios da confiança no médico ${ }^{5,14,22,27,29}$ e das questões relativas à medicação ${ }^{20,24,29}$. Os menores escores foram nos domínios preocupação com sigilo $5,20,22,24,29$ e preocupação financeira ${ }^{14,27}$.

Os estudos que utilizaram o HAT-QOL apontam que diferentes fatores sociodemográficos são responsáveis pela melhor ou pior avaliação da QV, como sexo, idade, cor branca, escolaridade, renda, tempo de diagnóstico, bem como pela prática de atividade física e sexualidade. Assim, concluem que as condições de vida e de saúde afetam diretamente a avaliação da QV, que pode ser impactada por meio de políticas públicas com vistas à inclusão social, respeitando as diferenças e compreendendo as especificidades da cronicidade do viver com HIV/AIDS 5,14,20,22,24,27,29. 
Os maiores escores apresentados nos artigos que utilizaram o WHOQOL foram nos domínios psicológico ${ }^{15,17,23,25,30,32}$ e ERCP ${ }^{3,13,16,21,28,31}$. Os menores escores foram nos domínios meio ambiente $\mathrm{e}^{3,13,16-18,21,28,30-32}$ e nível de independência ${ }^{23,25}$.

Os estudos que utilizaram o WHOQOL revelaram que as condições de vida impactam diretamente na avaliação da QV de pessoas vivendo com HIV. Ainda, apontam que os aspectos psicossociais interferem negativamente na QV, principalmente, quando essas pessoas já passaram por situações de estigma e preconceito, quando possuem relacionamento homoafetivo, ou por enfrentarem doenças oportunistas. Em suma, os estudos reiteram a necessidade de compreensão da QV como um conceito ampliado e multifatorial, com estratégias que perpassam o setor saúde ${ }^{3,13,15-}$ $19,21,23,25-26,28,30-32$

O estudo que comparou a avaliação da QV mensurada pelo HAT-QOL e pelo WHOQOL sugeriu que ambos os instrumentos se comportam de modo análogo na avaliação da QV de pessoas vivendo com HIV, sendo reconhecidos como instrumentos válidos e confiáveis para a população brasileira ${ }^{18}$

\section{DISCUSSÃO}

As discussões sobre o construto da QV, no campo da saúde, não devem apenas contribuir para a reflexão e crítica, mas também, devem ser considerados como uma proposição instituída por discursos e práticas com a finalidade de desenvolver e aprimorar protocolos e políticas ${ }^{33}$. A compreensão da QV como resultado de percepções individuais que residem na experiência humana, implica também no conceito de necessidades humanas básicas, que precisa ser ampliado para uma discussão em uma dimensão menos biológica e normativa e mais social e universal ${ }^{34}$.

No contexto da AIDS, a preocupação com o conceito de QV refere-se a um movimento dentro das ciências humanas e biológicas no sentido de valorizar parâmetros mais amplos que apenas o controle dos sintomas, a redução da mortalidade ou o aumento da expectativa de vida35. Dessa forma, ao discutir a QV de forma ampliada, é necessário compreendê-la como um processo dinâmico e mutável, que inclui as interações contínuas entre o indivíduo e o ambiente, portanto, as condições socioeconômicas, demográficas, culturais, psicológicas e espirituais, constituem-se fatores intrínsecos na avaliação da QV. Os baixos níveis de escolaridade e renda remetem às condições precárias de vida, vulnerabilidades sociais, culturais e econômicas, dificuldades no acesso aos serviços de saúde e a falta de conhecimento sobre seu estado de saúde e sobre a terapêutica, refletindo assim, negativamente na avaliação da QV.

No contexto das vulnerabilidades individuais e sociais dos indivíduos ao HIV, revestem-se de importância as estratégias e políticas sociais que visam à garantia de direitos e a inclusão desses grupos sociais na sociedade de forma autônoma e com respeito às diferenças.

Apesar da relação entre saúde e QV datar dos primórdios da medicina social, séculos XVIII e XIX ${ }^{36}$, os estudos científicos que utilizaram o construto da QV no campo da saúde tiveram aumento importante a partir da década de 1990, diante da necessidade de compreender a QV e suas relações com as questões biológicas, sociais e culturais ${ }^{37}$.

Neste sentido, alguns instrumentos foram criados com a finalidade de aferição da complexidade que envolve a avalição da QV, seja a partir de instrumentos específicos para a área da saúde, sejam aqueles que utilizam a saúde como indicador interveniente para a $\mathrm{QV}^{36}$. Entre os instrumentos desenvolvidos, o WHOQOL, elaborado por um grupo pesquisadores especialistas em saúde da OMS, possibilita a comparação de dados em uma perspectiva internacional e transcultural. Posteriormente, foi adaptado para pessoas vivendo com HIV, sendo amplamente utilizado por pesquisadores brasileiros. Outro instrumento, o HIV/AIDS Quality of Life (HAT-QOL), foi construído com o intuito de avaliar especificamente a QV de pessoas vivendo com HIV e as características e impactos da epidemia da AIDS para a QV desses indivíduos. Embora existam outros instrumentos, esses obtiveram maior destaque nas pesquisas brasileiras.

Em relação aos domínios de avaliação da QV apresentados pelos instrumentos, no domínio físico observa-se uma discordância entre os estudos que utilizaram o WHOQOL-HIV bref. Maiores escores neste domínio podem relacionar-se com a evolução do tratamento para o HIV/AIDS e a TARV, o que possibilitou não só o aumento da sobrevida, mas também a redução de infecções oportunistas, bem como de internações, o que pode influenciar na melhor avaliação da $\mathrm{QV}^{13}$. As medidas terapêuticas propiciam ao indivíduo a diminuição da sintomatologia, promovem a manutenção da capacidade funcional, além de favorecer a autoimagem, proporcionando as pessoas vivendo com HIV uma aparência "saudável", influenciando em sua autoestima e nas outras dimensões da QV24,26.

Pode-se inferir que estudos com menores escores neste domínio identificaram alterações provenientes do HIV na capacidade funcional, sintomatologia relacionada ao HIV ou efeitos colaterais relacionados à TARV. A TARV pode gerar efeitos colaterais, como náuseas, vômitos e a síndrome lipodistrófica, que pode ocasionar alterações físicas perceptíveis, e possível efeitos nas dimensões psicológicas e sociais da QV38. Outros fatores percebidos como negativos para esta dimensão é a presença de doenças oportunistas, altos níveis de carga viral e baixos níveis de linfócito T CD4+ ${ }^{23}$. 
Identificaram-se ainda menores escores no domínio meio ambiente em estudos que utilizaram o WHOQOL-HIV bref $^{13,16}$. As possíveis implicações estão relacionadas às vulnerabilidades e desigualdades sociais descritas. Além disso, enquanto agravo em saúde, a infecção pelo HIV provoca reflexos para a saúde da população, impondo a necessidade de terapêuticas e novos hábitos, o que gera aumento de custos para este grupo social. Este dado também emerge nas pesquisas que utilizaram o HAT-QOL ${ }^{5,14}$, evidenciada no domínio preocupação financeira.

Neste sentido, outra faceta que implica para o domínio meio ambiente é a inserção das pessoas vivendo com HIV nos serviços de saúde. No Brasil, o Programa IST/AIDS é considerado como referência mundial na distribuição da TARV e tratamento à esta população. Alguns estudos demonstram uma avaliação positiva dos serviços de atendimento especializados em HIV/AIDS pela clientela, avaliação ancorada nas relações com os profissionais de saúde, principalmente o médico, e na distribuição gratuita dos medicamentos ${ }^{17,38}$.

A confiança no médico ${ }^{5,14}$ foi um dos domínios com maiores escores, demonstrando a importância deste profissional no tratamento das pessoas vivendo com HIV. Relações interpessoais positivas e com confiança entre médico e paciente favorecem a adesão e a aceitação do indivíduo a sua condição sorológica e ao tratamento prescrito ${ }^{14}$.

O domínio questões relativas à medicação, avaliado pelo HAT-QOL, obteve o segundo maior escore, dentre os artigos selecionados 5 . A TARV é considerada um fator essencial na manutenção da QV das pessoas vivendo com HIV, por propiciar a redução e/ou controle dos sintomas, prolongamento dos estágios latentes da infecção e afastamento da possibilidade do adoecimento ${ }^{13}$. O tratamento medicamentoso pode influir tanto de forma positiva na QV dos indivíduos, quanto também de forma negativa, por gerar a necessidade de mudanças de hábitos, como a tomada regular dos antirretrovirais e a adaptação da rotina das pessoas vivendo com HIV ${ }^{5,15}$.

O domínio que apresentou menores escores no HAT-QOL foi a preocupação com o sigilo ${ }^{5,14}$. Este fato se associa com os impactos sociais que o HIV/AIDS trazem para a vida dos indivíduos. Pesquisas ${ }^{5,14,23}$ apontam o estigma e a discriminação enquanto fator relevante e negativo para a QV das pessoas vivendo com HIV. Estigma e atitudes discriminatórias geram sentimentos como angústia, ansiedade e medo, ocasionando impactos na dimensão psicológica da QV e a diminuição da autoestima, além da adoção de comportamentos retraídos e do isolamento social para ocultamento do diagnóstico. Essa omissão ocorre em todos os níveis das relações sociais, fazendo com que as pessoas vivendo com HIV assumam um comportamento tenso e de retração, no intuito de não demonstrar qualquer fator que possa revelar a sorologia positiva ao $\mathrm{HIV}^{5,20,22,24}$. Este comportamento, além de influenciar no domínio das relações sociais, pode impactar na adesão e aceitação do sujeito ao tratamento.

No combate ao estigma e discriminação, estudos ${ }^{5,14,26}$ apontam como grande estratégia de redução de danos as diversas redes sociais de inserção das pessoas vivendo com HIV, cujo apoio social, confiança e empatia se estabeleçam para auxiliar no enfrentamento das questões inerentes ao HIV, além de favorecer a adesão e fortalecer os vínculos sociais, promovendo o empoderamento das pessoas vivendo com HIV, enquanto ator social.

Portanto, estudos que visam à discussão e atualização das questões do viver e conviver com o HIV/AIDS se fazem sempre atuais e oportunos. Isto porque, a avaliação da QV, sendo uma construção individual e social, simultaneamente, recebe influencias das condições de saúde atuais de vida das pessoas vivendo com HIV - presença de sintomas, efeitos adversos ou infecções oportunistas -, bem como do momento vivenciado no âmbito das políticas de saúde e políticas sociais, como o acesso aos serviços de saúde, exames e medicamentos, oportunidades de emprego, condições de moradia, transporte, entre outros. Destarte, a QV das pessoas vivendo com HIV deve ser constantemente avaliada e os impactos, tanto negativos quanto positivos, devem servir de indicadores para a reformulação das políticas públicas e melhorias nas condições de vida.

\section{CONSIDERAÇÕES FINAIS}

Estudos que busquem conhecer os fatores que intervém na QV de pessoas vivendo com HIV, como pesquisas com o construto da QV revestem-se de significados, por favorecer a identificação dos aspectos que interferem na QV e favorecer a construção de estratégias com vistas a redução dos danos para esse grupo social. Configuram-se como essenciais as políticas e ações que visem a redução das desigualdades e vulnerabilidades de grupos populacionais e que podem interferir nas ações de prevenção, combate e tratamento ao HIV.

Os instrumentos para aferição da QV proporcionam meios para captar o bem-estar e a percepção dos sujeitos sobre sua vida. Ressalta-se, porém, que estes instrumentos são construídos por meios quantitativos, onde domínios diferentes possuem a mesma pontuação, o que pode gerar uma falsa visão de uma QV eminentemente positiva e/ou negativa. Os estudos selecionados enquanto amostra desta revisão integrativa possuem as médias majoritariamente positivas, entretanto, observaram-se domínios com escores de avaliação negativa e outros com médias máximas de avaliação, gerando uma média final tendendo a uma avaliação de QV positiva. A QV é um construto multidimensional, subjetivo e individual, com interligação entre seus domínios. Assim, a construção de uma análise que busca a 
identificação de todos os fatores que influenciam a QV de forma individual e suas relações promove uma melhor compreensão da QV e favorece na elaboração de estratégias com vistas a domínios específicos e com influência final para a QV das pessoas vivendo com HIV.

\section{REFERÊNCIAS}

1. UnAIDS. Programa Conjunto das Nações Unidas sobre HIV/AIDS no Brasil [internet]. Estatísticas. [cited 2020 jun 12]; Availabre from: https://unAIDS.org.br/estatisticas/

2. Ministério da Saúde (BR). Secretaria de Vigilância em Saúde. Departamento de Doenças de Condições Crônicas e Infecções Sexualmente Transmissíveis. Boletim epidemiológico HIV/AIDS 2019. Brasília: Ministério da Saúde; 2019. [cited 2020 jun 12 ]; Available from: http://www.aids.gov.br/pt-br/pub/2019/boletim-epidemiologico-de-hivaids-2019

3. Silva RAR, Duarte FHS, Nelson ARC, Holanda RR. AIDS epidemic in Brazil: analysis of current profile. Rev. Enferm. UFPE [Internet] 2013 [cited 2020 Jun 12]; 7(10):6039-48. Available from: https://periodicos.ufpe.br/revistas/revistaenfermagem/article/view/12233

4. Rocha GSA, Angelim RCM, Andrade ARL, Aquino JA, Abrão FMS, Costa AM. Nursing care of HIV-positive patients: considerations in the light of phenomenology. Rev. Min. Enferm. [Internet] 2015 [cited 2018 Jan 10]; 19(2):258-61. DOI: http://www.dx.doi.org/10.5935/1415-2762.20150040

5. Reis RK, Santos CB, Dantas RAS, Gir E. Quality of life, sociodemografic factors and sexuality of people living with HIV/AIDS. Texto contexto Enferm. [Internet] 2011 [cited 2018 jan 10]; 20(3): 565-75. DOI: http://dx.doi.org/10.1590/S010407072011000300019

6. Oliveira FBM, Moura MEB, Araujo, TME, Andrade EMLR. Quality of life and associated factors in people living with HIV/AIDS. Acta Paul. Enferm. [Internet] 2016 [cited 2017 Dec 08]; 6:510-16. DOI: http://dx.doi.org/10.1590/1982-0194201500086

7. World Health Organization (WHO), World Health Organization Quality of Life Group (Whoqol). The World Health Organization quality of life assessment - WHOQOL: position paper from the World Health Organization. Soc. Sci. Med. [Internet] 1995 [cited 2018 Dec 13]; 41:1403-10. DOI: https://doi.org/10.1016/0277-9536(95)00112-K

8. Costa TL, Oliveira DC, Formozo GA. Quality of life and AIDS from the perspective of persons living with HIV: a preliminar contribution by the structural approach to social representations. Cad. Saúde Pública [Internet] 2015 [cited 2018 Dec 08]; 2:365-76. DOI: http://dx.doi.org/10.1590/0102-311X00180613

9. Hipolito RL, Oliveira DC, Gomes AMT, Costa TL. Social representations of quality of life in HIV/AIDS: the role of time since diagnosis. Rev. enferm. UERJ [Internet] 2014 [cited 2018 Dec 08]; 22:753-9. DOI: http://dx.doi.org/10.12957/reuerj.2014.12840

10. Almeida MAB, Gutierrez GL, Marques R. Qualidade de vida: definição, conceitos e interfaces com outras áreas de pesquisa. São Paulo: EACH/USP; 2012

11. Cecilio HPM, Oliveira DC. Revisão integrativa como método de pesquisa em enfermagem: uma sistematização. In: Silva Neto BR (org). Ciências da Saúde: Da Teoria à Prática 3. Ponta Grossa, PR: Atena Editora. 2019; 208-223.

12. Moher D, Liberati A, Tetzlaff J, Altman DG, The PRISMA Group. Galvão TF, Pansani TSA (trad). Principais itens para relatar revisões sistemáticas e meta-análises: a recomedação PRISMA. Epidemiol. Serv. Saúde [Internet] 2015 [cited 2020 Jul 14]; 24(2). DOI: https://doi.org/10.5123/S1679-49742015000200017

13. Gaspar J, Reis RK, Pereira FMV, Neves LAS, Castrighini CC, Gir E. Quality of life in women with HIV/AIDS in a municipality In the state of São Paulo. Rev. Esc. Enferm. USP [Internet] 2011 [cited 2018 Jan 10]; 45(1): 230-6. DOI: http://dx.doi.org/10.1590/S0080-62342011000100032

14. Lopes PSD, Silva MMG, Torres IC, Stadñik CMB. Quality of life in HIV-positive patients over 50 years of age. Revista da AMRIGS [Internet] 2011 [cited 2018 Jan 12]; 55(4): 356-360. Available from: https://pesquisa.bvsalud.org/portal/resource/pt/biblio835384 ?lang=es

15. Ferreira BE, Oliveira IM, Paniago AMM. Quality of life of people living with HIV/AIDS and its relationship with CD4+ lymphocytes, viral load and time of diagnosis. Rev. Bras. Epidemiol. [Internet] 2012 [cited 2018 Jan 12]; 15(1): 75-84. DOI: https://doi.org/10.1590/S1415-790X2012000100007

16. Medeiros B, Saldanha AAW. The relationship between religiousness and quality of life in people living with HIV. Estudos de Psicologia [Internet] 2012 [cited 2018 Dec 20]; 29(1): 53-61. DOI: http://dx.doi.org/10.1590/S0103-166X2012000100006

17. Medeiros B, Silva J, Saldanha AAW. Biopsychosocial determinants that predict quality of life in people living with HIV/AIDS. Estudos de Psicologia [Internet] 2013 [cited 2018 Jan 12]; 18(4):543-550. DOI: https://doi.org/10.1590/S1413$294 \times 2013000400001$

18. Borba KB, Bertoni RF, Fratoni KRBP, Silva J, Traebert J. Comparative study of the performance of WHOQOL-HIV-Bref and HIV/AIDS-target quality of life in the quality of life evaluation of individuals living with HIV/AIDS. Arq. Catarin. Med. [Internet] 2014 [cited 2018 Jan 16]; 43(3): 26-31. Available from: http://www.acm.org.br/revista/pdf/artigos/1295.pdf

19. Oliveira Filho JS, Silva PE, Freitas FFQ, Soares JP, Costa MAG, Silva ACO. Quality of life of people living with hiv/aids. Rev. Baiana de Enf. [Internet] 2014 [cited 2018 Sep 18]; 28(1):61-8. Available from: https://portalseer.ufba.br/index.php/enfermagem/article/view/9088

20. Okuno MFP, Gomes AC, Meazzini L, Scherrer Júnior G, Belasco Junior D, Belasco AGS. Quality of life in elderly patients living with HIV/AIDS. Cad. Saúde Pública [Internet] 2014 [cited 2018 Jan 16]; 30(7):1551-9. DOI: https://doi.org/10.1590/0102$311 \times 00095613$

21. Bellini JM, Reis RK, Reinato LAF, Magalhães RLB, Gir E. Quality of life of HIV seropositive women. Acta Paul. Enferm. [Internet] 2015 [cited 2018 Jan 16]; 28(4): 350-4. DOI: https://doi.org/10.1590/1982-0194201500059 
22. Galvão MT, Soares LL, Pedrosa SC, Fiuza ML, Lemos LA. Quality of life and adherence to antiretroviral medication in people with HIV. Acta Paul. Enferm. [Internet] 2015 [cited 2018 jan 16]; 28(1):48-53. DOI: https://doi.org/10.1590/1982-0194201500009

23. Oliveira FBM, Moura MEB, Araújo TME, Andrade EMLR. Quality of life and associated factors in people living with HIV/AIDS. Acta Paul. Enferm. [Internet] 2015 [cited 2018 Jan 16]; 28(6):510-6. DOI: https://doi.org/10.1590/1982-0194201500086

24. Okuno MFP, Gonsuen GC, Campanharo CRV, Fram DS, Batista REA, Belasco AGS. Quality of life, socioeconomic profile, knowledge and attitude toward sexuality from the perspectives of individuals living with Human Immunodeficiency Virus. Rev. Latino-am. Enferm. [Internet] 2015 [cited 2018 Jan 16]; 23(2):192-9. DOI: https://doi.org/10.1590/0104-1169.3424.2542

25. Cunha GH, Fiuza MLT, Gir E, Aquino OS, Pinheiro AKB, Galvão MTG. Quality of life of men with AIDS and the model of social determinants of health. Ver. Latino-am. Enferm. [Internet] 2015 [cited 2018 Set 10]; 23(2):183-91. DOI: http://dx.doi.org/10.1590/0104-1169.0120.2541

26. Teixeira M, Mendes MT, Borba KP, Pereira EM, Borba E. Evaluation of the quality of women living with hiv' life. Rev. Enferm. UFSM [Internet] 2015 [cited 2018 Jan 16]; 5(2): 360-7. DOI: https://doi.org/10.5902/2179769215277

27. Soares GB, Garbin CAS, Rovida TAS, Garbin AJl. Quality of life of people living with HIV/AIDS treated by the specialized service in Vitória-ES, Brazil. Cienc. Saúde Colet. [Internet] 2015 [cited 2018 Jan 16]; 20(4):1075-84. DOI: https://doi.org/10.1590/141381232015204.00522014

28. Hipolito RL, Oliveira DC, Costa TL, Marques SC, Pereira ER, Gomes AMT. Quality of life of people living with HIV/AIDS: temporal, socio-demographic and perceived health relationship. Ver. Latino-am. Enferm. [Internet] 2017 [cited 2018 Sep 20]; 25:e2874. DOI: http://dx.doi.org/10.1590/1518-8345.1258.2874

29. Caliari JS, Reinato LAF, Pio DPM, Lopes LP, Reis RK, Gir E. Quality of life of elderly people living with HIV/AIDS in outpatient follow-up. Rev. Bras. Enferm. [Internet] 2018 [cited 2020 Jul 10]; 71(Suppl 1):513-22. DOI: https://doi.org/10.1590/0034-71672017-0127

30. Cecilio HPM, Oliveira DC, Oliveira DS, Domingues JP, Marques SC. Quality of life of people living with HIV. Cienc. Cuid. Saude [Internet] 2018 [cited 2020 Jul 10]; 17(4): e45032. DOI: https://doi.org/10.4025/cienccuidsaude.v17i4.45032

31. Silveira MF, Ferreira AC, Brito MFSF, Pinho L, Teixeira Júnior AL, Carneiro M. Psychometric properties of the WHOQOL-HIV Bref for quality of life assessment. Psico-USF [Internet] 2019 [cited 2020 Jul 10]; 24(3):475-87. DOI: https://doi.org/10.1590/141382712019240306

32. Cecilio HPM, Oliveira DS, Marques SC, Apostolidis T, Oliveira DC. Quality of life of people living with HIV treated in public health services. Rev. enferm. UERJ [Internet] 2018 [cited 2020 Jul 10]; 26:e37461. DOI: https://doi.org/10.12957/reuerj.2019.37461

33. Matta GC. A medida política da vida: a invenção do WHOQOL e a construção de políticas de saúde globais [Doctoral dissertation]. Rio de Janeiro: Universidade do Estado do Rio de Janeiro; 2005. [cited 2020 Jul 10]; Available from: http://bdtd.ibict.br/vufind/Record/UERJ_073a937eeb262a744cc2d7bee0ac82d1

34. Oliveira DC. A Enfermagem e as necessidades humanas básicas: o saber/fazer a partir das representações sociais [Full Professor]. Rio de Janeiro: Universidade do Estado do Rio de Janeiro; 2001.

35. Fleck MPA, Leal OF, Louzada S, Xavier M, Chachamovich E, Vieira G, Santos L, Pinzon V. Development of the Portuguese version of the OMS evaluation instrument of quality of life. Rev. Bras. Psiquiatr. [Internet] 1999 [cited 2018 Sep 18]; 21(1):19-28. DOI: http://dx.doi.org/10.1590/S1516-44461999000100006

36. Minayo MCS, Hartz ZMA, Buss PM. Quality of life and health: a necessary debate. Ciênc. Saúde Colet. [Internet] 2000 [cited 2018 Dec 15]; 5(1):7-18. DOI: http://dx.doi.org/10.1590/S1413-81232000000100002

37. Gordia AP, Quadros TMB, Oliveira MTC, Campos W. Quality of life: historical context, definition, assessment and associated factors. R. bras. Qual. Vida [Internet] 2011 [cited 2018 Jan 12]; 3(1): 40-52. DOI: https://dx.doi.org/10.3895/S217508582011000100005

38. Paschoal EP, Espírito-Santo CC, Gomes AMT, Santos, EI, Oliveira DC, Pontes APM. Adherence to antiretroviral therapy and its representations for people living with HIV/AIDS. Esc. Anna Nery [Internet] 2014 [cited 2018 Jan 12]; 18(1): 32-40. DOI: http://dx.doi.org/10.5935/1414-8145.20140005 